09

\title{
Модифицированная теория связанных волн и численный метод для анализа брэгговских зеркал с произвольным профилем показателя преломления
}

\author{
(C) Р.А. Мастеров, С.Ю. Карпов \\ ООО „Софт-Импакт“, \\ 194044 Санкт-Петербург, Россия \\ e-mail: roman.masterov@str-soft.com
}

Поступило в Редакцию 7 июля 2021 г.

В окончательной редакции 7 июля 2021 г.

Принято к публикации 11 августа 2021 г.

\begin{abstract}
Развиты аналитический и эффективный численный подходы к анализу оптических характеристик брэгговских зеркал с произвольным профилем показателя преломления. Полученные с помощью модифицированной теории связанных волн аналитические выражения для коэффициентов отражения/прохождения света от/через брэгговское зеркало с высокой точностью предсказывают основные его характеристики как при низком, так и при высоком контрасте показателя преломления. Гибридный численный метод включает в себя численный расчет матрицы переноса на одном периоде зеркала и ее аналитическое мультиплицирование для произвольного числа периодов. Развитые методы применены к анализу свойств практически важных брэгговских зеркал, изготовленных на основе напыляемых пар диэлектриков $\mathrm{Ta}_{2} \mathrm{O}_{5} / \mathrm{SiO}_{2}$ и эпитаксиальных нитридов и арсенидов III-группы. Детально рассмотрены характеристики зеркал с плавными (градиентными) интерфейсами между отдельными слоями.
\end{abstract}

Ключевые слова: вертикально излучающие лазеры, коэффициент отражения, полоса отражения, модифицированная теория связанных волн, численное моделирование.

DOI: 10.21883/JTF.2021.12.51766.209-21

\section{Введение}

Благодаря высокой эффективности, низкой потребляемой мощности, высокой скорости модуляции, круговой форме лазерного пучка, легко достигаемому одномодовому режиму генерации и возможности интеграции в двумерную матрицу, лазеры с вертикальным выводом излучения (или вертикально излучающие лазеры (ВИЛ); в английской аббревиатуре - VCSELs) получили широкое развитие в последнюю декаду. Первоначально сфера их применения ограничивалась передачей данных в волоконно-оптических сетях и оптических соединениях в центрах обработки данных и суперкомпьютерах [1]. Затем ВИЛ стали интенсивно использоваться в качестве источников направленного излучения в сенсорах, лазерных принтерах и компьютерных мышках $[1,2]$. И, хотя в дальнейшем потребности в ВИЛ на рынке коммуникаций только росли, со временем эти приборы открывали все новые области приложений. В частности, запросы безопасности и социального общения в интернете потребовали создания систем быстрого распознавания лиц, где ВИЛ выступали в качестве компактных источников сканирующего света $[3,4]$. Такие лазеры нашли также широкое применение в компактных проекторах, формирующих $3 D$-изображение для кинотеатров и компьютерных игр [5], а одномодовая генерация света оказалась незаменимой для создания атомных часов [6] в системах позиционирования и синхронизации времени в различных приборах и устройствах. В биологии ВИЛ служат элементами биофлуоресцентных датчиков для диагностики рака на ранней стадии и скрининга наркотиков [7]. ВИЛ позволили существенно уменьшить размеры устройств биофлуоресцентного анализа, сделать их портативными, снижая тем самым стоимость проводимых исследований. Особые преимущества ВИЛ имеют в скоростном детектировании газообразного аммиака, позволяя с высоким временным разрешением оценивать концентрации реагентов и промежуточных продуктов непосредственно в реакционной камере без отбора проб и с возможностью повторной калибровки [8]. Наконец, матрицы ВИЛ необходимы для создания лидаров, т.е. систем обнаружения и определения дальности объектов с помощью света [9].

Впервые идея создания ВИЛ появилась в 1977 году применительно к системе материалов $\mathrm{AlGaAs} / \mathrm{GaAs}$, обеспечивающей длину волны генерации лазера в районе $850 \mathrm{~nm}[1,10]$. Однако первая экспериментальная реализация этой идеи была осуществлена в системе материалов GaInAsP/InP на длине волны $\lambda=1300 \mathrm{~nm}$ [11], соответствующей одному из окон прозрачности оптического волокна. В дальнейшем исследования ВИЛ в этих системах материалов развивались параллельно, хотя более технологичная система $\mathrm{AlGaAs} / \mathrm{GaAs}$ и превалировала. Использование InGaAs активной области позволило, с одной стороны, уменьшить пороговые токи ВИЛ, а с другой - задействовать длину волны 
генерации $980 \mathrm{~nm}$ [10]. Для расширения спектрального диапазона ВИЛ в сторону более коротких длин волн $(\lambda=780 \mathrm{~nm})$ была сначала использована та же система $\mathrm{AlGaAs} / \mathrm{GaAs}$ [12], а затем система материалов $\mathrm{AlGaInP} / \mathrm{GaAs}(\lambda=670 \mathrm{~nm})$ [13], развитие которой стимулировалось разработкой красных светодиодов. Освоение сине-фиолетового $(\lambda=405-450 \mathrm{~nm})$, а затем зеленого $(\lambda=500-525 \mathrm{~nm})$ диапазонов видимого спектра обусловлено развитием технологии нитридов III группы, т. е. системы материалов $\mathrm{AlGaInN} / \mathrm{Al}_{2} \mathrm{O}_{3}$ [14]. Дальнейшее расширение рабочих спектральных областей ВИЛ связываются с нитридной системой (в область ультрафиолетового излучения) [15] и системой материалов $\mathrm{AlGaInAsSb} / \mathrm{GaSb}$ (в инфракрасный спектральный диапазон) [16].

Существенным элементом конструкции ВИЛ являются брэгговские зеркала (Б3), образующие высокодобротный резонатор лазера. Оптимизация БЗ представляет собой сложную многофакторную задачу. Ее главная цель - получение максимально высокого коэффициента отражения света, генерируемого лазером. Из-за возможных флуктуаций толщин и показателей преломления слоев, составляющих БЗ, необходимо добиваться высокого коэффициента отражения при минимальном числе слоев. Для этого следует выбирать пары материалов с высоким контрастом показателя преломления. Однако на практике этот контраст оказывается существенно разным в случае внутренних Б3, полученных чередованием эпитаксиальных полупроводниковых слоев, или же внешних, сформированных напылением диэлектрических пленок. Поэтому оптимизация числа пар слоев, их толщин и используемых материалов, определяющих показатели преломления, является первостепенной задачей.

Однако использование эпитаксиальных БЗ с высоким контрастом показателя преломления дает слишком большое последовательное сопротивление ВИЛ из-за образования вблизи резких гетерограниц Б3 высокоомных областей объемного заряда. Для уменьшения сопротивления было предложено использовать плавные интерфейсы и модулированное легирование слоев Б3 [17-20]. Но использование слоев с плавным изменением состава материала, как правило, приводит к уменьшению эффективности дифракции света в БЗ и, как следствие, к падению его отражательной способности, что требует дополнительной оптимизации конструкции Б3. Для этого необходимо использовать эффективные методы анализа оптических свойств БЗ, работающие при произвольном профиле показателя преломления.

Использование БЗ с плавными интерфейсами в нитридных ВИЛ обсуждалось в [21] в контексте их влияния на коэффициент отражения БЗ. Однако в этом случае ситуация осложняется еще и тем, что на границах пар слоев $\mathrm{AlGaN} / \mathrm{GaN}$ или $\mathrm{AlInN} / \mathrm{GaN}$, из которых обычно изготавливаются Б3, появляются поляризационные заряды [22], которые могут существенно изменить проводимость БЗ как в позитивном, так и в негативном направлении в зависимости от знака заряда. Из-за этого плавные интерфейсы применяются на практике лишь на одном (чаще на верхнем $[23,24]$ ) интерфейсе, внося асимметрию в распределении показателя преломления. И, наконец, упругие напряжения в БЗ, вызванные рассогласованием постоянных решетки $[25,26]$, существенно сужают возможность выбора пар материалов с высоким контрастом показателя преломления, а также приводят к необходимости введения дополнительных технологических слоев [26,27], усложняющих распределение показателя преломления на одном периоде БЗ.

Таким образом, налицо необходимость разработки эффективных и оперативных методов анализа оптических свойств БЗ с произвольным распределением показателя преломления по периоду. Целью настоящей работы является разработка эффективной аналитической модели Б3, позволяющей оперативно оптимизировать его конструкцию и получать распределения интенсивности света внутри Б3, необходимое для оценки оптических потерь, а также разработка гибридного численного подхода, соединяющего в себе численное решение уравнения Гельмгольца на одном периоде Б3, с матричным методом, позволяющим анализировать свойства всего многопериодного БЗ. Эти подходы используются далее для анализа Б3 с различными профилями показателя преломления.

\section{1. Теория}

Рассмотрим Б3, представляющее собой среду с периодически изменяющейся диэлектрической проницаемостью $\varepsilon(z+a)=\varepsilon_{r}(z)+i \varepsilon_{i}(z)$, включающую в себя в общем случае вещественную $\varepsilon_{r}$ и мнимую $\varepsilon_{i}$ части, где $a-$ период структуры (рис. 1). Обычно $\varepsilon_{r}(z) \gg \varepsilon_{i}(z)$, и тогда мнимая часть $\varepsilon_{i}(z)=\alpha(z) \varepsilon_{r}^{1 / 2}(z) / k$ выражается через коэффициент поглощения света $\alpha$, а вещественная часть $\varepsilon_{r}(z)=n^{2}(z)$ - через показатель преломления $n$. БЗ расположено между средами с показателями преломления $n_{i}$ и $n_{t}$. Падающая волна с амплитудой $A_{i}$ приходит к БЗ слева из среды с показателем преломления $n_{i}$, и в эту же среду уходит отраженная волна с амплитудой $A_{r}$.

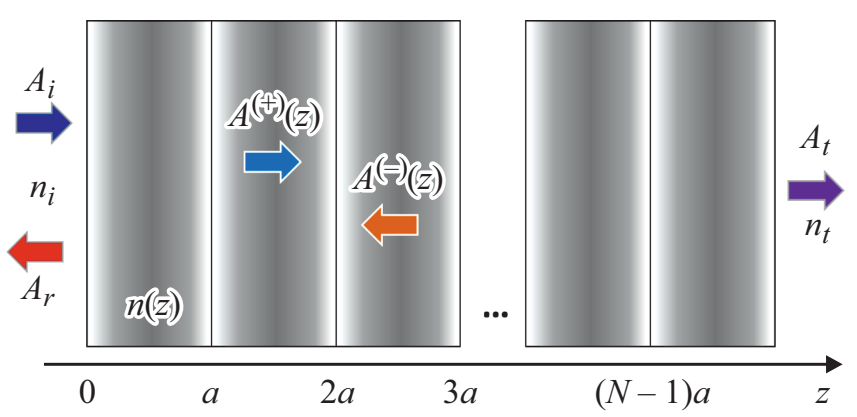

Рис. 1. Схематическая структура брэгговского зеркала, амплитуды прямой $A^{(+)}(z)$ и обратной $A^{(-)}(z)$ волн в структуре, а также амплитуды падающей $A_{i}$, отраженной $A_{r}$ и прошедшей $A_{t}$ волн. 
Прошедшая через Б3 волна с амплитудой $A_{t}$ уходит в среду с показателем преломления $n_{t}$ (рис. 1).

В $1 D$-приближении электромагнитная волна описывается уравнением Гельмгольца относительно амплитуды электрического поля $E(z, t)$ :

$$
\frac{d^{2} E(z)}{d z^{2}}+k^{2} \varepsilon(z) E(z)=0, \quad E(z, t)=E(z) \exp (-i \omega t)
$$

где $z$ - координата, направленная по нормали к слоям Б3, $k=2 \pi / \lambda-$ волновой вектор, $\lambda$ - длина волны света в вакууме. Магнитное поле световой волны выражается через электрическое поле как $H(z)=(i / k) d E(z) / d z$.

В качестве граничных условий на внешних и внутренних интерфейсах БЗ выступает непрерывность электрического и магнитного поля, что означает непрерывность $E$ и производной $d E / d z$.

\section{1. Модифицированная теория связанных волн}

Исходно теория связанных волн предложена Когельником и Шенком [28] для анализа периодических диэлектрических структур. Она применима, если амплитуда модуляции диэлектрической проницаемости $\Delta \varepsilon$ мала по сравнению с ее средним значением $\varepsilon_{0}: \Delta \varepsilon / \varepsilon_{0} \ll(\pi m)^{-1}$, где $m$ - порядок дифракции, на который настроено Б3 [29]. Отсюда также видно, что применимость подхода Когельника и Шенка ограничивается в том числе низкими порядками дифракции.

Однако в случае наиболее часто используемых Б3 из чередующихся пар слоев с большим контрастом показателя преломления погрешность предсказаний этой теории становится достаточно большой. В [30] была предложена модифицированная теория связанных волн (МТСВ) со значительно более широкой областью применимости, где осуществляется частичный учет многоволновой дифракции света, особенно в случае высоких порядков дифракции, за счет изменения выражений для коэффициентов связи. Учет многоволновой дифракции позволяет расширить область применения МТCВ, которую формально можно представить как $\Delta \varepsilon / \varepsilon_{0} \ll(\pi m)^{-1}+\pi m$ [29]. Кроме того, МТСВ хорошо работает как в случае непрерывно изменяющейся, так и в случае разрывной диэлектрической проницаемости. Таким образом, именно МТСВ оказывается оптимальной для построения аналитической теории распространения света в Б3.

Согласно [30], решение уравнения (1) ищется в форме

$$
\begin{aligned}
E(z)= & \frac{1}{\varepsilon^{1 / 4}(z)}\left\{A^{(+)}(z) \exp [i \psi(z)]\right. \\
& \left.+A^{(-)}(z) \exp [-i \psi(z)]\right\}
\end{aligned}
$$

где фаза

$$
\psi(z)=k \int_{0}^{z} \varepsilon^{1 / 2}\left(z^{\prime}\right) d z^{\prime} \cong k \int_{0}^{z} n\left(z^{\prime}\right) d z^{\prime}+\frac{1}{2} i \int_{0}^{z} \alpha\left(z^{\prime}\right) d z^{\prime},
$$

а $A^{(+)}(z)$ и $A^{(-)}(z)$ - суть медленно изменяющиеся амплитуды прямой и обратной волн в Б3. Решение основных уравнений МТСВ [30] дает следующие общие выражения для этих амплитуд:

$$
\begin{gathered}
A^{(+)}(z)=C_{1} \exp \left(-\gamma_{-} z\right)+C_{2} \exp \left(\gamma_{+} z\right) \\
A^{(-)}(z)=C_{1} r_{B}^{\infty} \exp \left(-\gamma_{+} z\right)-\frac{\kappa_{-n}^{(+)}}{\kappa_{n}^{(-)} r_{B}^{\infty}} C_{2} \exp \left(\gamma_{-} z\right) .
\end{gathered}
$$

Здесь $\gamma_{ \pm}=\gamma \pm\left(\frac{1}{2} \alpha_{0}-i \delta\right), \quad \gamma=\left[\left(\frac{1}{2} \alpha_{0}-\delta\right)^{2}+\right.$ $\left.+\kappa_{-m}^{(+)} \kappa_{m}^{(-)}\right]^{1 / 2}-$ комплексное волновое число, такое, что $\operatorname{Re} \gamma \geq 0, n_{0}=a^{-1} \int_{0}^{a} n(z) d z$ и $\alpha_{0}=a^{-1} \int_{0}^{a} \alpha(z) d z$ усредненные по периоду показатель преломления и коэффициент оптических потерь Б3, $\delta=k n_{0}-$ $-(\pi / a) m-$ отстройка от брэгговского резонанса $m$-го порядка, $C_{1}$ и $C_{2}$ - произвольные постоянные, а параметр $r_{B}^{\infty}=-\kappa_{-m}^{(+)} /\left(\gamma+\frac{1}{2} \alpha_{0}-i \delta\right)$ представляет собой амплитудный коэффициент отражения света от полубесконечного БЗ [29].

Коэффициенты связи $\kappa_{m}^{( \pm)}$прямой и обратной волн, соответствующие $m$-му порядку дифракции, определяются в МТСВ выражениями

$$
\begin{aligned}
& \kappa_{m}^{( \pm)}=\frac{1}{a}\left\{\int _ { 0 } ^ { a } \frac { d z } { 4 \varepsilon } \frac { d \varepsilon } { d z } \operatorname { e x p } \left[2 i \left( \pm \psi(z) m\left(k n_{0}+\frac{1}{2} i \alpha_{0}\right) z\right.\right.\right. \\
& \left.\left.-\frac{\pi}{a} m z\right)\right]+\frac{1}{4} \sum_{j=1}^{p} \ln \frac{\varepsilon\left(z_{j}+0\right)}{\varepsilon\left(z_{j}-0\right)} \exp [2 i \\
& \left.\left.\times\left( \pm \psi\left(z_{j}\right) m\left(k n_{0}+\frac{1}{2} i \alpha_{0}\right) z_{j}-\frac{\pi}{a} m z_{j}\right)\right]\right\}
\end{aligned}
$$

где интеграл понимается в смысле главного значения, второй член представляет собой выделенный в явном виде вклад от интерфейсов с разрывной диэлектрической проницаемостью, а $z_{j}(j=1,2 \ldots p)$ - суть координаты этих интерфейсов.

Граничные условия для уравнения Гельмгольца при $z=0$ и $L$, предполагающие непрерывность тангенциальных составляющих электрического и магнитного полей, дают четыре соотношения, которые при заданной амплитуде $A_{i}$ позволяют найти постоянные $C_{1}$ и $C_{2}$, амплитуды прошедшей $\left(A_{t}\right)$ и отраженной $\left(A_{r}\right)$ волн, а также амплитудные коэффициенты отражения

$$
r_{\Sigma}^{L}=\frac{r_{i}+\tilde{r}}{1+r_{i} \tilde{r}}, \quad \tilde{r}=\frac{r_{B}^{L}+Y_{B} r_{t} \exp \left(2 i \varphi_{t}\right)}{1+r_{B}^{L}\left(\kappa_{m}^{(-)} / \kappa_{m}^{(+)}\right) r_{t} \exp \left(2 i \varphi_{t}\right)},
$$




$$
\begin{gathered}
r_{i}=\frac{n_{i}-\varepsilon_{r}^{1 / 2}(0)}{n_{i}+\varepsilon_{r}^{1 / 2}(0)}, \quad r_{t}=\frac{\varepsilon_{r}^{1 / 2}(L)-n_{t}}{\varepsilon_{r}^{1 / 2}(L)+n_{t}}, \\
r_{B}^{L}=\frac{-\kappa_{-m}^{(+)} \operatorname{sh}(\gamma L)}{\gamma \operatorname{ch}(\gamma L)+\left(\frac{1}{2} \alpha_{0}-i \delta\right) \operatorname{sh}(\gamma L)}, \\
Y_{B}=\frac{\gamma \operatorname{ch}(\gamma L)-\left(\frac{1}{2} \alpha_{0}-i \delta\right) \operatorname{sh}(\gamma L)}{\gamma \operatorname{ch}(\gamma L)+\left(\frac{1}{2} \alpha_{0}-i \delta\right) \operatorname{sh}(\gamma L)}
\end{gathered}
$$

и коэффициент прохождения

$$
\begin{gathered}
t_{\Sigma}^{L}=\frac{\left(1+r_{i}\right)\left(1+r_{t}\right)}{1+r_{i} \tilde{r}} \cdot \frac{t_{B}^{L}}{1+r_{B}^{L}\left(\kappa_{m}^{(-)} / \kappa_{-m}^{(+)}\right) r_{t} \exp \left(2 i \varphi_{t}\right)} \\
t_{B}^{L}=\frac{\varepsilon^{1 / 4}(0)}{\varepsilon^{1 / 4}(L)} \cdot \frac{\gamma \exp \left(i \varphi_{t}\right)}{\gamma \operatorname{ch}(\gamma L)+\left(\frac{1}{2} \alpha_{0}-i \delta\right) \operatorname{sh}(\gamma L)}
\end{gathered}
$$

света через БЗ.Тогда энергетические коэффициенты отражения $\left(R_{B}^{L}\right)$ и прохождения $\left(T_{B}^{L}\right)$ от/через Б3 суть

$$
R_{B}^{L}=\left|r_{\Sigma}^{L}\right|^{2} \quad \text { и } \quad T_{B}^{L}=\left(n_{t} / n_{i}\right)\left|t_{\Sigma}^{L}\right|^{2} .
$$

В отсутствие поглощения света в брэгговском зеркале выполняется соотношение $R_{B}^{L}+T_{B}^{L}=1$. Распределение интенсивности света в брэгговском зеркале задается прямым вычислением $|E(z)|^{2}$ с использованием выражения (2).

\section{2. Численный метод расчета}

Для расчета характеристик Б3, выполненных из чередующихся пар слоев с постоянными диэлектрическими проницаемостями внутри каждого слоя, обычно используется эффективный метод матриц переноса [31]. Согласно этому методу, амплитуды прямой и обратной волн в произвольной точке $z=z_{0}$ связаны с амплитудами этих волн в точке $z=z_{0}+a$ матричным соотношением

$$
\left[\begin{array}{l}
A^{(+)\left(z_{0}\right)} \\
A^{(-)\left(z_{0}\right)}
\end{array}\right]=\left[\begin{array}{ll}
m_{11} & m_{12} \\
m_{21} & m_{22}
\end{array}\right] \cdot\left[\begin{array}{l}
A^{(+)}\left(z_{0}+a\right) \\
A^{(-)\left(z_{0}+a\right)}
\end{array}\right],
$$

где $m_{i k}(i, k=1,2)$ - комплексные элементы матрицы переноса, имеющие аналитическую форму в случае пар слоев с постоянными диэлектрическими проницаемостями [31]. В силу того, что матрицы переноса являются унимодулярными, можно непосредственно получить связь амплитуд прямой и обратной волн на противоположных концах Б3, содержащего $N$ пар слоев:

$$
\begin{gathered}
{\left[\begin{array}{l}
A^{(+)(0)} \\
A^{(-)(0)}
\end{array}\right]=\left[\begin{array}{ll}
M_{11} & M_{12} \\
M_{21} & M_{22}
\end{array}\right] \cdot\left[\begin{array}{l}
A^{(+)(L)} \\
A^{(-)(L)}
\end{array}\right], \beta=\frac{1}{2}\left(m_{11}+m_{22}\right),} \\
M_{11}=m_{11} \mathscr{U}_{N-1}(\beta)-\mathscr{U}_{N-2}(\beta), \quad M_{12}=m_{12} \mathscr{U}_{N-1}(\beta), \\
M_{21}=m_{21} \mathscr{U}_{N-1}(\beta) \quad M_{22}=m_{22} \mathscr{U}_{N-1}(\beta)-\mathscr{U}_{N-2}(\beta),
\end{gathered}
$$

где $\mathscr{U}_{N}(\beta)$ - полином Чебышева 2-го рода, для вычисления которого имеются хорошо известные рекуррентные соотношения.
В случае Б3 со сложным профилем диэлектрической проницаемости элементы матрицы переноса не могут быть получены аналитически. Чтобы сохранить в целом преимущества матричного метода при произвольном распределении диэлектрической проницаемости на периоде Б3, уравнение Гельмгольца решалось численно на одном периоде, из этого решения извлекались элементы матрицы $m_{i k}$, а затем использовалось соотношение (9) для получения связи амплитуд прямой и обратной волн на разных концах БЗ.

Зная элементы $M_{i k}$ полной матрицы переноса, из граничных условий можно получить амплитудные коэффициенты отражения

$$
r_{\Sigma}^{L}=\frac{r_{i}+\tilde{r}}{1+r_{i} \tilde{r}} ; \quad \tilde{r}=\frac{M_{21}+M_{22} r_{t}}{M_{11}+M_{12} r_{t}}
$$

и прохождения

$$
t_{\Sigma}^{L}=\frac{\left(1+r_{i}\right)\left(1+r_{t}\right)}{\left(1+r_{i} \tilde{r}\right)\left(M_{11}+M_{12} r_{t}\right)}
$$

света от/через БЗ. Здесь $r_{i}$ и $r_{t}$ - амплитудные коэффициенты френелевского отражения света от первого и последнего интерфейсов БЗ соответственно. Энергетические коэффициенты отражения по-прежнему рассчитываются по формулам (7).

\section{2. Результаты}

\section{1. Брэгговские зеркала с постоянными показателями преломления слоев}

В качестве примера расчета были выбраны Б3 типичных структур ВИЛ, изготовленных на основе нитридов III группы [32,33]. Одно из таких Б3 было выполнено из 50 пар слоев $\mathrm{Al}_{0.18} \mathrm{In}_{0.82} \mathrm{~N} / \mathrm{GaN}$, настроенных на длину волны света $\lambda_{0}=410 \mathrm{~nm}$, с последним $\mathrm{GaN}$-слоем. Другое Б3 включало в себя 35 пар слоев $\mathrm{Al}_{0.15} \mathrm{In}_{0.85} \mathrm{~N} / \mathrm{Al}_{0.2} \mathrm{Ga}_{0.8} \mathrm{~N}$, настроенных на длину волны $\lambda_{0}=343 \mathrm{~nm}$, с последним $\mathrm{Al}_{0.2} \mathrm{Ga}_{0.8} \mathrm{~N}$-слоем. Эти Б3, входящие в эпитаксиальные структуры лазеров и обычно выращиваемые на толстых GaN-слоях, должны обеспечивать максимально полное отражение генерируемого света. Для сравнения с результатами характеризации таких Б3, представленных в [32,33], предполагалось, что падающий на них свет приходит из воздуха. В качестве другого примера Б3, выводящего свет из лазера, была выбрана типичная структура из пар слоев $\mathrm{Ta}_{2} \mathrm{O}_{5} / \mathrm{SiO}_{2}$, настроенных на длину волны $410 \mathrm{~nm}$, с последним $\mathrm{SiO}_{2}$-слоем. Предполагалось, что такая многослойная структура наносится с помощью напыления $\mathrm{Ta}_{2} \mathrm{O}_{5}$ и $\mathrm{SiO}_{2}$ на $\mathrm{GaN}$, а свет, проходя через нее, выводится в воздух. Показатели преломления используемых в БЗ материалов, приведенные в таблице, были взяты из [34,35] (в случае нитридов III группы в качестве таковых использовались показатели преломления обыкновенных 
Характеристики материалов брэгговских зеркал. Среды, из которых свет падает на зеркало, и среды, в которые свет выводится

\begin{tabular}{c|c|c|c}
\hline \multirow{2}{*}{ Брэггоское зеркало } & Материал & $\begin{array}{c}\text { Показатель } \\
\text { преломления }\end{array}$ & $\begin{array}{c}\text { Показатели } \\
\text { преломления } \\
n_{i} / n_{t}\end{array}$ \\
\hline \multirow{2}{*}{$50 \cdot\left(\mathrm{Al}_{0.18} \mathrm{In}_{0.82} \mathrm{~N} / \mathrm{GaN}\right)$} & $\mathrm{GaN}$ & 2.53 & $1.0 / 2.53$ \\
\hline & $\mathrm{Al}_{0.18} \mathrm{In}_{0.82} \mathrm{~N}$ & 2.28 & \\
\cline { 2 - 3 } & $\mathrm{Al}_{0.2} \mathrm{Ga}_{0.8} \mathrm{~N}$ & 2.61 & \multirow{2}{*}{$1.0 / 2.71$} \\
\cline { 2 - 4 } & $\mathrm{Al}_{0.15} \mathrm{In}_{0.85} \mathrm{~N}$ & 2.345 & $2.53 / 1.0$ \\
\hline
\end{tabular}
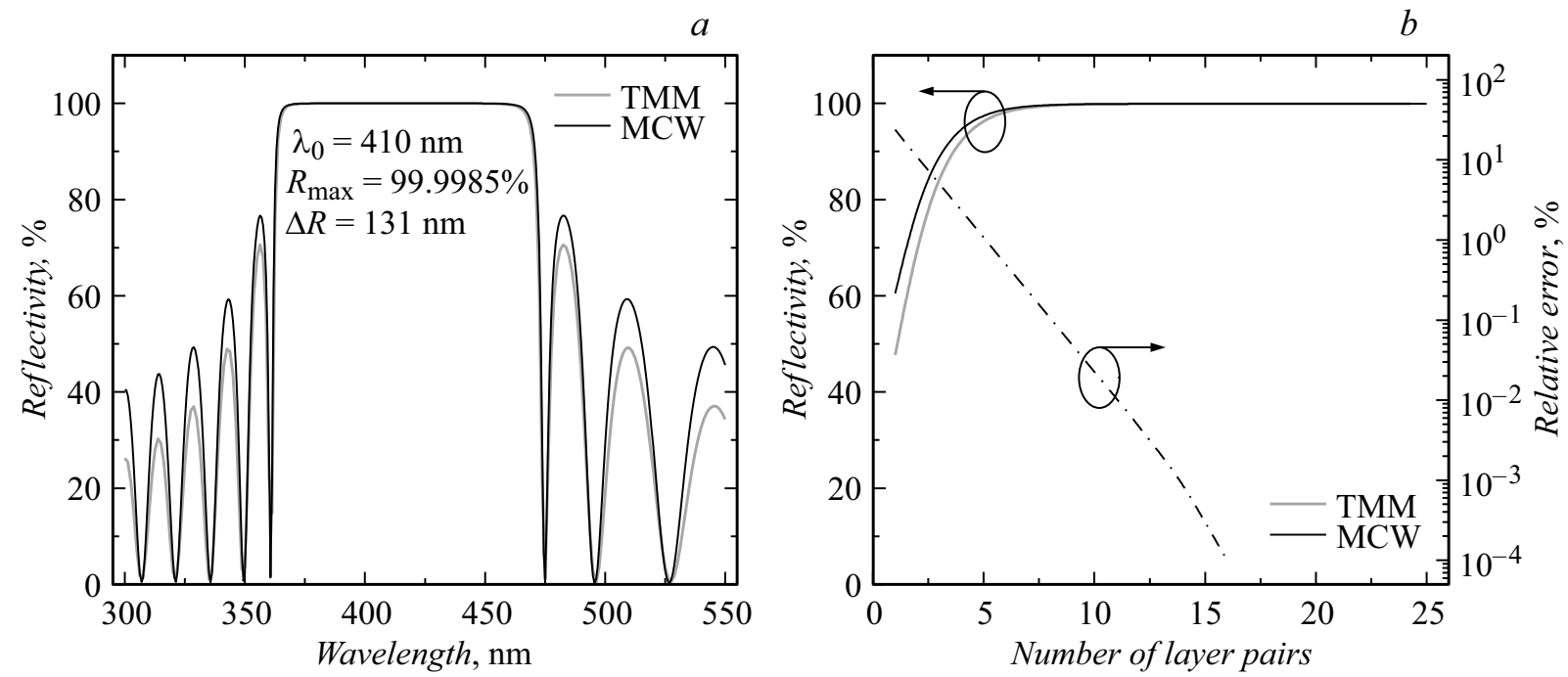

Рис. 2. Спектральная зависимость энергетического коэффициента отражения $\mathrm{Ta}_{2} \mathrm{O}_{5} / \mathrm{SiO}_{2}$ Б3 (a) и зависимость коэффициента отражения на длине волны $410 \mathrm{~nm}$ от числа пар слоев $(b)$. Черная сплошная линия соответствует расчетам по модифицированной теории связанных волн (MCW), а серая - точным результатам, полученным методом матриц переноса (ТММ); штрих-пунктирная линия показывает относительную погрешность расчета по теории связанных волн.

волн). Для получения максимального коэффициента отражения света при минимальном числе слоев БЗ толщины этих слоев $d_{1}$ и $d_{2}$ выбирались равными четверти длины волны света в веществе, т.е. $d_{1,2}=\frac{1}{4} \lambda_{0} / n_{1,2}$, где $n_{1}$ и $n_{2}$ - показатели преломления пар слоев Б3 [31].

На рис. 2 показаны спектральные зависимости энергетического коэффициента отражения $\mathrm{Ta}_{2} \mathrm{O}_{5} / \mathrm{SiO}_{2}$ Б3 с 15 парами слоев, полученные с помощью МТСВ (черная линия) и методом матриц переноса [31] (серая линия), дающим в данном случае точное решение задачи. В расчетах мы пренебрегали дисперсией показателей преломления обоих диэлектриков. Видно, что в пределах полосы отражения Б3 оба метода с графической точностью дают практически неразличимые результаты, несмотря на высокий контраст показателей преломления $\mathrm{Ta}_{2} \mathrm{O}_{5}$ и $\mathrm{SiO}_{2}$. Столь же хорошо предсказывается ширина полосы отражения на полувысоте: $\Delta R=131 \mathrm{~nm}$. При этом погрешность предсказания первых максимумов коэффициента отражения за пределами этой полосы не превышает $10 \%$.
О поведении погрешности предсказаний МТСВ лучше всего судить, анализируя зависимость максимального значения коэффициента отражения Б3 (на длине волны $410 \mathrm{~nm}$ ) от числа пар слоев $\mathrm{Ta}_{2} \mathrm{O}_{5}$ и $\mathrm{SiO}_{2}$ (рис. 2,b). Поскольку МТСВ разработана специально для периодических структур, ожидалось, что она будет плохо работать при малом числе пар. Однако оказалось, что уже для двух пар слоев погрешность в определении коэффициента отражения методом МТСВ становится $\sim 10 \%$, а при пяти парах падает до $\sim 1 \%$, уменьшаясь далее экспоненциально с числом пар слоев. Таким образом, МТСВ можно использовать для предсказания величины коэффициента отражения БЗ и ширины полосы отражения с достаточно высокой точностью.

$\mathrm{C}$ точки зрения самих характеристик $\mathrm{Ta}_{2} \mathrm{O}_{5} / \mathrm{SiO}_{2}$ Б3, рис. 2, $b$ показывает, что максимальный коэффициент отражения достигается практически уже при 10 парах слоев $\mathrm{Ta}_{2} \mathrm{O}_{5}$ и $\mathrm{SiO}_{2}$. Интересно, что расчеты показывают увеличение этого числа слоев до 15-16 при падении 

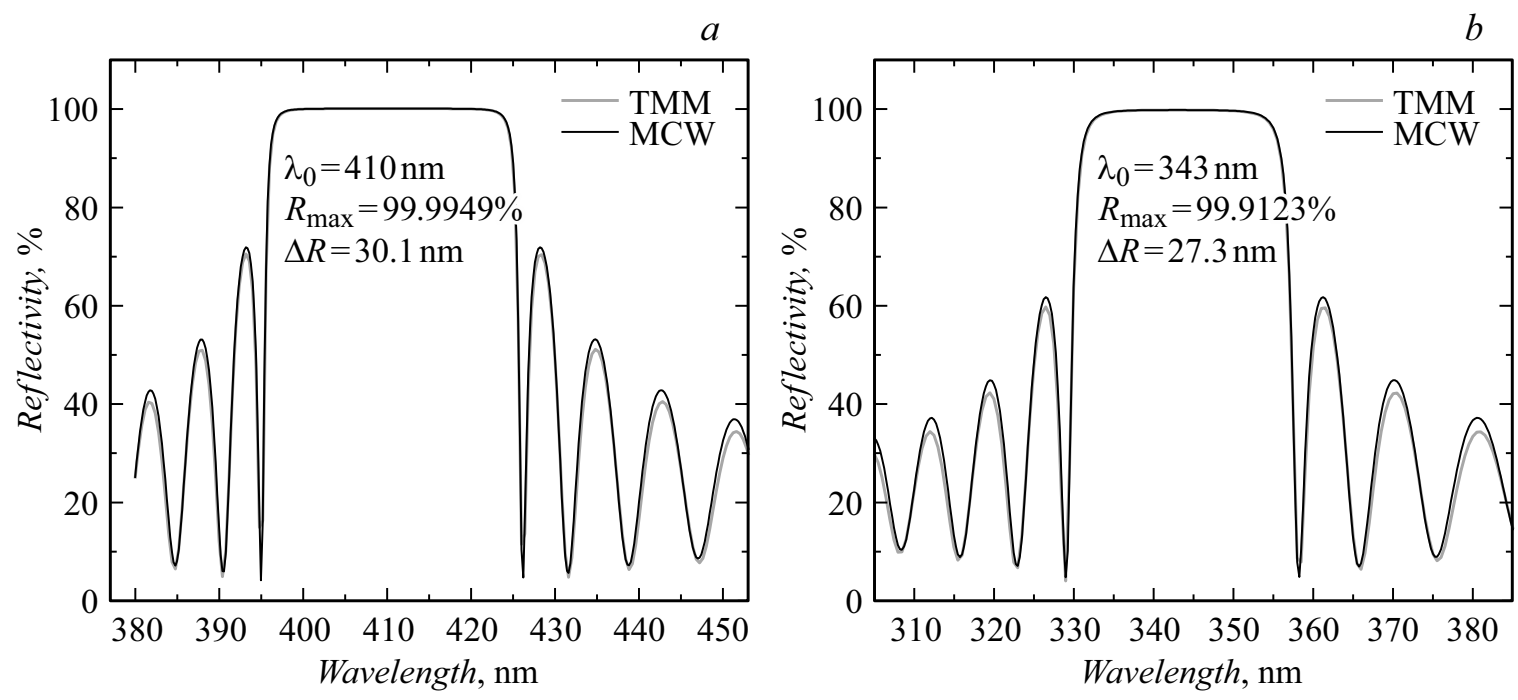

Рис. 3. Спектральные зависимости энергетических коэффициентов отражения $\mathrm{Al}_{0.18} \mathrm{In}_{0.82} \mathrm{~N} / \mathrm{GaN} \quad(a) \quad$ и $\mathrm{Al}_{0.15} \mathrm{In}_{0.85} \mathrm{~N} / \mathrm{Al}_{0.2} \mathrm{Ga}_{0.8} \mathrm{~N}(b)$ Б3. Черная сплошная линия соответствует расчетам по модифицированной теории связанных волн $(\mathrm{MCW})$, а серая - точным результатам, полученным методом матриц переноса (TMM).

света на БЗ из воздуха. Именно такое число слоев $\mathrm{Ta}_{2} \mathrm{O}_{5}$ и $\mathrm{SiO}_{2}$ и используется на практике [36,37].

На рис. 3 приведены спектральные зависимости энергетических коэффициентов отражения $\mathrm{Al}_{0.18} \mathrm{In}_{0.82} \mathrm{~N} / \mathrm{GaN} \quad$ (a) $\quad$ и $\quad \mathrm{Al}_{0.15} \mathrm{In}_{0.85} \mathrm{~N} / \mathrm{Al}_{0.2} \mathrm{Ga}_{0.8} \mathrm{~N}$ Б3, рассчитанные с помощью МТСВ (черная линия) и с помощью матриц переноса (серая линия). Сравнение результатов расчета этими двумя методами показывает небольшую разницу между величинами коэффициента отражения в широком спектральном диапазоне. Это связано с относительно малым контрастом показателей преломления составляющих Б3 полупроводников. Максимальные значения коэффициентов отражения составили 99.9949\% и 99.9123\%, а полуширины полос отражения - 30.1 и $27.3 \mathrm{~nm}$ для $\mathrm{Al}_{0.18} \mathrm{In}_{0.82} \mathrm{~N} / \mathrm{GaN}$ и $\quad \mathrm{Al}_{0.15} \mathrm{In}_{0.85} \mathrm{~N} / \mathrm{Al}_{0.2} \mathrm{Ga}_{0.8} \mathrm{~N} \quad$ Б3 соответственно. Экспериментально на этих Б3, выращенных методом МОС-гидридной эпитаксии, были получены следующие коэффициенты отражения и полуширины полос отражения: $99.7 \%$ и $30 \mathrm{~nm}$ для длины волны $410 \mathrm{~nm}$ и $99.5 \%$ и $20 \mathrm{~nm}$ для длины волны $343 \mathrm{~nm}$. Как видно из сравнения с теоретическими предсказаниями, существенное расхождение наблюдается только у полуширины полосы отражения $\mathrm{Al}_{0.15} \mathrm{In}_{0.85} \mathrm{~N} / \mathrm{Al}_{0.2} \mathrm{Ga}_{0.8} \mathrm{~N}$ Б3. На наш взгляд, оно может быть связано с флуктуациями толщин слоев этого Б3, возникшими в процессе его выращивания. Другой возможной причиной являются упругие напряжения, возникающие в структуре при ее выращивании на GaN-буферном слое, приводящие к изгибу эпитаксиальной структуры в целом.

При анализе ВИЛ часто оказывается востребованным распределение интенсивности света внутри БЗ. Такие распределения, соответствующие длине волны света $410 \mathrm{~nm}$, показаны на рис. 4 для $\mathrm{Ta}_{2} \mathrm{O}_{5} / \mathrm{SiO}_{2}$ и
$\mathrm{Al}_{0.18} \mathrm{In}_{0.82} \mathrm{~N} / \mathrm{GaN}$ брэгговских зеркал. Видно, что распределение интенсивности, полученное с помощью МТСВ, испытывает нефизические разрывы, связанные с прямым включением в амплитуды диэлектрической проницаемости $\varepsilon^{-1 / 4}(z)$ (см. выражение $(2)$ ), которая сама в рассмотренных нами случаях разрывна. Обратной стороной этого недостатка является достаточно точное воспроизведение профиля интенсивности света вдали от разрывов. Кроме того, при уменьшении контраста показателя преломления в БЗ наличие разрывов становится малосущественным (рис. $4, b)$.

\section{2. Оптические свойства БЗ с градиентными интерфейсами}

Для уменьшения электрического сопротивления Б3, выполненных на основе тройных полупроводниковых твердых растворов, обычно используются профили состава с плавным (градиентным) переходом от широкозонного материала, имеющего меньший показатель преломления, к узкозонному, имеющему больший показатель преломления [17-20]. В этом случае оценка оптических характеристик Б3 и оптимизация их конструкции, включая число периодов, нужное для реализации высокого коэффициента отражения, становится нетривиальной задачей. В данном разделе для решения этой задачи используется МТСВ. Из-за отсутствия точного решения уравнений Максвелла для произвольного профиля показателя преломления, результаты, полученные с помощью МТСВ, сравниваются с численными расчетами, выполненными методом, описанным в разд. 2.2.

Далеко не все твердые растворы позволяют получить с помощью эпитаксии Б3 с произвольным профилем состава. Прежде всего это связано с генерацией упругих 


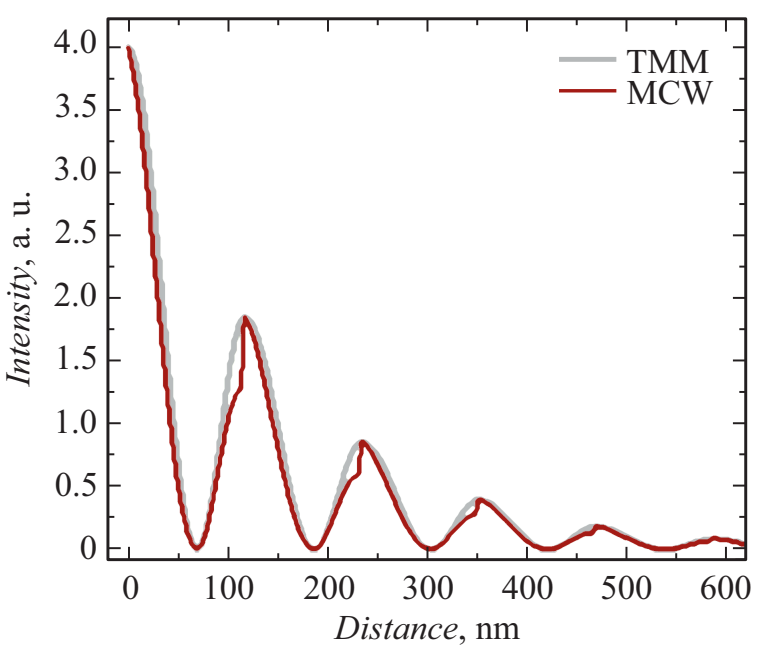

$b$

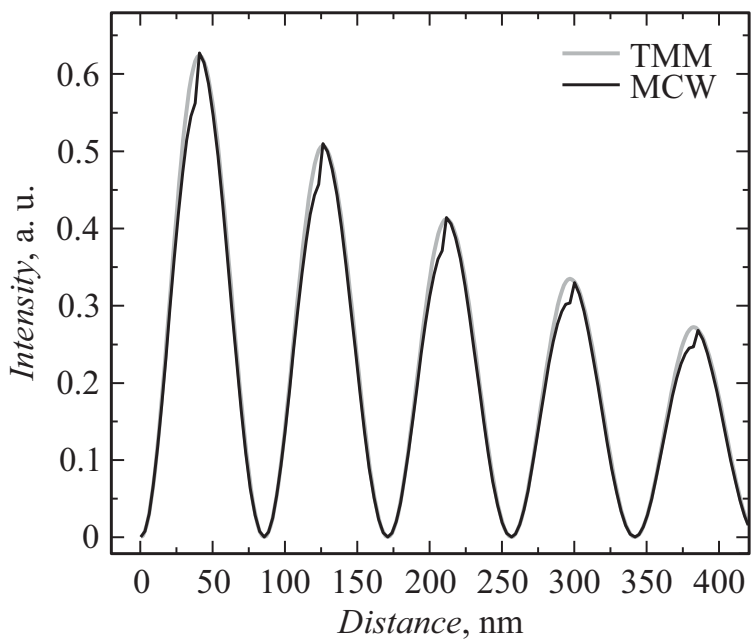

Рис. 4. Распределения интенсивности света внутри $\mathrm{Ta}_{2} \mathrm{O}_{5} / \mathrm{SiO}_{2}(a)$ и $\mathrm{Al}_{0.18} \mathrm{In}_{0.82} \mathrm{~N} / \mathrm{GaN}(b)$ брэгговских зеркал на длине волны $410 \mathrm{~nm}$. Черная сплошная линия соответствует расчетам по модифицированной теории связанных волн (MCW), а серая - точным результатам, полученным методом матриц переноса (ТММ).

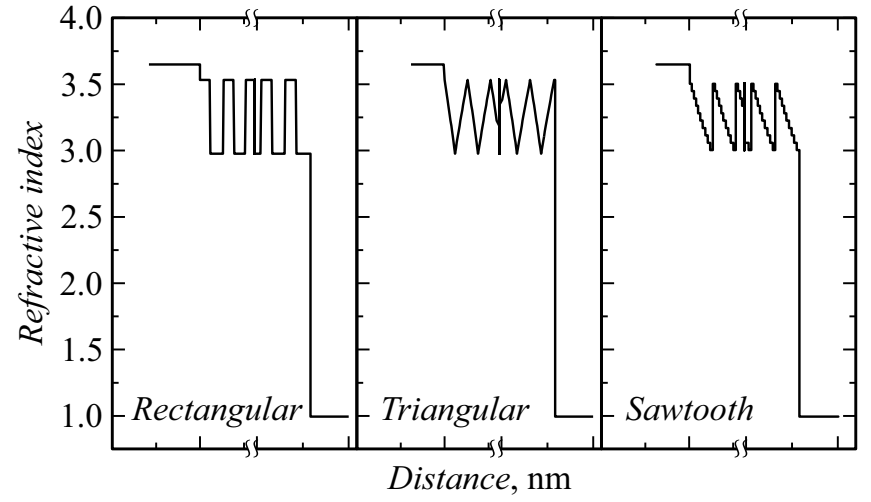

Рис. 5. Профили показателя преломления в брэгговских зеркалах, анализируемые в настоящей работе.

напряжений в эпитаксиальных структурах из-за рассогласования постоянных кристаллической решетки бинарных составляющих твердых растворов. Это проблема отсутствует в твердых растворах $\mathrm{AlGaAs,} \mathrm{согласованных}$ по постоянной решетки с GaAs во всем диапазоне изменения состава. По этой причине в качестве практически значимых примеров нами были выбраны Б3 с треугольным и пилообразным профилями показателя преломления (рис. 5). Минимальное и максимальное значения показателя преломления были выбраны при этом соответствующими твердым растворам $\mathrm{Al}_{0.92} \mathrm{Ga}_{0.08} \mathrm{As}$ и $\mathrm{Al}_{0.16} \mathrm{Ga}_{0.84} \mathrm{As}$.

Каждое БЗ считалось состоящим из 25 периодов. Предполагалось, что свет приходит на Б3 из слоя GaAs, а выходит в воздух. Результирующие профили показателя преломления задачи в целом представлены на рис. 5 . Там же для сравнения показан прямоугольный профиль показателя преломления, включающий на одном пери- оде четвертьволновые (для длины волны $\lambda_{0}=850 \mathrm{~nm}$ ) слои $\mathrm{Al}_{0.92} \mathrm{Ga}_{0.08} \mathrm{As}$ и $\mathrm{Al}_{0.16} \mathrm{Ga}_{0.84} \mathrm{As}$ толщиной 71.36 и $60.15 \mathrm{~nm}$ соответственно. Периоды всех трех Б3 были выбраны одинаковыми.

На рис. 6, $a, b$ приведены спектральные зависимости коэффициентов отражения Б3 с треугольным и пилообразным профилем показателя преломления соответственно, полученные с помощью МТСВ (черные линии) и численным методом (серые линии). Видно, что максимумы коэффициентов отражения в обоих методах заметно смещены в длинноволновую сторону относительно длины волны света $\lambda_{0}=850 \mathrm{~nm}$, на которую настроено БЗ с прямоугольным показателем преломления. Смещение различно для МТСВ и численного метода; это связано с тем, что МТСВ оперирует с квазиклассической фазой распространяющейся волны $\psi(z)$ (см. выражение (3)), а численный метод находит фазу непосредственно из уравнения Гельмгольца. А наличие красного смещения, предсказываемого обоими методами, показывает, что точная настройка БЗ на нужную длину волны не может базироваться на оценках, выработанных для прямоугольного профиля показателя преломления, но требует детальных расчетов и основанных на них коррекций периода Б3.

Максимальные значения коэффициентов отражения Б3 как с треугольным, так и с пилообразным профилем оказываются меньшими, чем у Б3 с прямоугольным профилем показателя преломления. Это особенно хорошо видно на рис. $6, c$ и $d$, показывающим зависимости коэффициентов отражения на длине волны $\lambda_{0}=850 \mathrm{~nm}$ от числа периодов БЗ. Видно, что в случае прямоугольного профиля коэффициент отражения выходит на насыщение уже примерно при 15 периодах, в то время как для треугольного и пилообразного профилей для этого требуются 25 периодов и более. Это означает, 

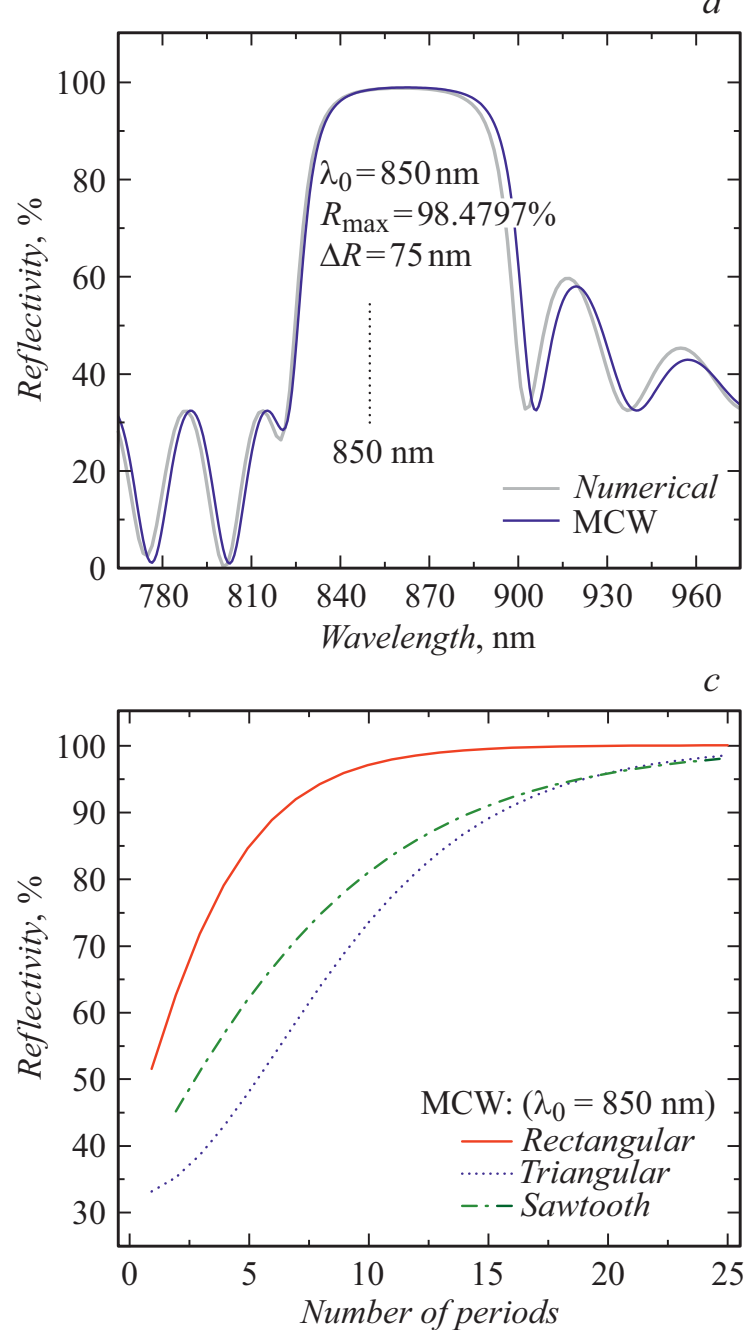
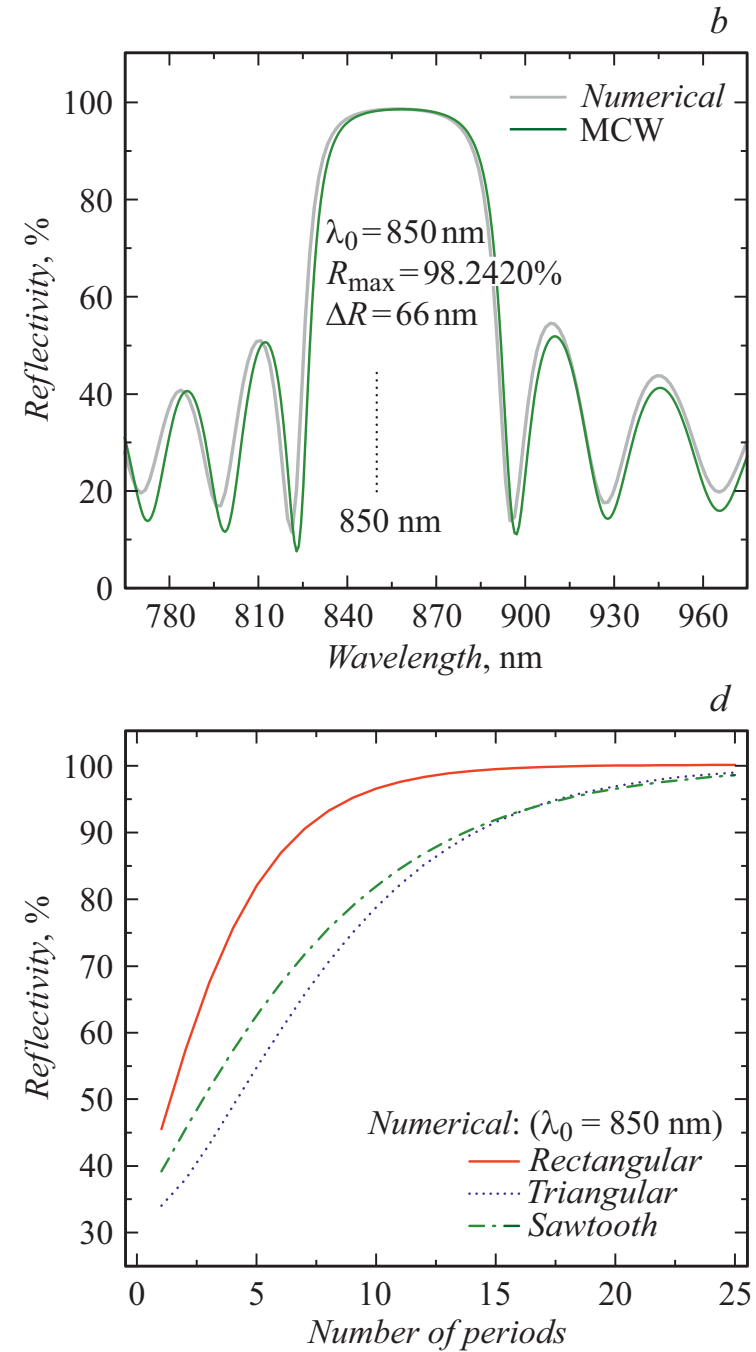

Рис. 6. Спектральные зависимости энергетических коэффициентов отражения от $\mathrm{Al}_{0.92} \mathrm{Ga}_{0.08} \mathrm{As}_{2} / \mathrm{Al}_{0.16} \mathrm{Ga}_{0.84} \mathrm{As}$ Б3 с треугольным $(a)$ и пилообразным $(b)$ профилем показателя преломления и 25 периодами. Зависимость коэффициента отражения таких Б3 на длине волны света $850 \mathrm{~nm}$ от числа периодов, полученная с помощью МТСВ $(c)$ и численным методом $(d)$; здесь результаты для прямоугольного профиля преломления также включены для сравнения.

что снижение сопротивления БЗ за счет использования плавных интерфейсов требует тщательной оптимизации числа его периодов. Причина влияния профиля показателя преломления на коэффициент отражения легко интерпретируется в терминах константы связи, введенной в МТСВ. Действительно, абсолютная величина константы связи $\left|\kappa_{m}^{( \pm)}\right|$для прямоугольного профиля показателя преломления $\left(12996 \mathrm{~cm}^{-1}\right)$ оказывается в разы выше, чем у пилообразного $\left(6412 \mathrm{~cm}^{-1}\right)$ или треугольного $\left(8181 \mathrm{~cm}^{-1}\right)$ профилей. Поскольку именно константа связи определяет, в конечном счете, и максимальный коэффициент отражения, и ширину полосы отражения, влияние на эту константу профиля показателя преломления оказывается решающим для оптических характеристик БЗ.

Интересной особенностью спектральной зависимости коэффициента отражения, показанной на рис. $6, a$, явля- ется асимметрия ее плечей относительно длины волны, соответствующей максимуму отражения, и заметный уровень отражения справа от основной спектральной полосы. Специально проведенное моделирование показало, что эти особенности во многом определяются френелевским отражением света на выходном интерфейсе БЗ. Действительно, при 25 периодах коэффициент отражения БЗ с треугольным профилем показателя преломления только еще начинает выходить на насыщение, так что отражение света на выходном интерфейсе БЗ оказывается значимым. При согласовании показателей преломления на этом интерфейсе (здесь не показано) значения коэффициента отражения за пределами основной спектральной полосы понижаются, а характер асимметрии меняется, хотя до конца она не исчезает. На наш взгляд, последнее может быть связано с остаточным вкладом френелевского отражения на входном интерфейсе в общее отражение света от БЗ. 
Сравнивая результаты, полученные с помощью МТСВ и численным методом, следует заметить, что за исключением спектрального сдвига полосы отражения БЗ оба метода дают весьма близкие результаты как для максимального значения коэффициента отражения, так и для ширины этой полосы. Это происходит как в случае непрерывно изменяющегося показателя преломления (треугольный профиль), так и случае разрывнонепрерывного изменения (пилообразный профиль). Также близкими оказываются зависимости коэффициента отражения от числа периодов Б3 (ср. рис. 6, с и $d$ ). Отсутствие разрывов диэлектрической проницаемости у треугольного профиля позволяет избавиться от нефизичных разрывов в распределении интенсивности в БЗ, аналогичных описанным в разд. 2.1, однако при этом возникает небольшое отклонение значений интенсивности вблизи ее максимумов, предсказываемых с помощью МТСВ относительно полученных численно (здесь не показано). В целом это отклонение меньше, чем у разрывных профилей диэлектрической проницаемости, и поэтому малосущественно.

\section{Заключение}

Работа направлена на развитие эффективных методов анализа оптических характеристик Б3 с произвольным профилем показателя преломления, что важно для практической оптимизации их электрических параметров. На основе МТСВ, учитывающей многоволновую дифракцию света, получены аналитические выражения для коэффициентов отражения/прохождения света от/через Б3 с произвольным профилем показателя преломления. В качестве альтернативного подхода построен гибридный алгоритм численного расчета этих же коэффициентов. Данный алгоритм основывается на численном построении матрицы переноса на одном периоде Б3 с последующим аналитическим ее мультиплицированием для получения оптических характеристик зеркала с произвольным числом периодов. Такой подход позволяет избежать накопления погрешности в прямом численном расчете БЗ целиком и существенно ускоряет получение конечного результата.

На примере Б3 с прямоугольным профилем показателя преломления, допускающим точное решение, показано, что МТСВ с высокой точностью предсказывает такие важные характеристики Б3, как максимальное значение коэффициента отражения света и его спектральную зависимость, включая ширину полосы отражения на полувысоте. Этот же вывод подтвержден и для Б3 с плавными (градиентными) интерфейсами путем сравнения результатов, полученных с помощью МТСВ, и численного гибридного подхода.

Обычная теория связанных волн [28] хорошо работает при малом контрасте показателя преломления в Б3. Путем сравнения результатов с точным решением, допускаемым для прямоугольного профиля показателя преломления, показано, что МТСВ с высокой точностью предсказывает оптические характеристики Б3 как в случае малого, так и в случае большого контраста показателя преломления, что связано с более аккуратным учетом многоволновой дифракции в рамках этого метода $[29,30]$.

В качестве недостатков МТСВ следует отметить следующее:

1) нефизические разрывы в распределении интенсивности света внутри БЗ, возникающие из-за вхождения в выражение для интенсивности (2) разрывного распределения диэлектрической проницаемости.

2) сдвиг спектральной зависимости коэффициента отражения Б3 с плавными интерфейсами, полученной с помощью МТСВ, относительно рассчитанной численно. Этот сдвиг возникает из-за использования квазиклассической фазы распространяющейся волны, тогда как в численном методе фаза получается непосредственно из уравнения Гельмгольца. Однако его величина невелика - она составляет всего от 1 до 3\% ширины полосы отражения, что не является критичным для практических расчетов.

С учетом вышеизложенных выводов можно рекомендовать использовать МТСВ, в первую очередь для анализа максимального значения коэффициента отражения света от БЗ и ширины полосы отражения.

Сравнение результатов наших расчетов с экспериментальными данными по отражению света от $\mathrm{Al}_{0.18} \mathrm{In}_{0.82} \mathrm{~N} / \mathrm{GaN}$ и $\mathrm{Al}_{0.15} \mathrm{In}_{0.85} \mathrm{~N} / \mathrm{Al}_{0.2} \mathrm{Ga}_{0.8} \mathrm{~N}$ Б3 показало в целом их хорошее согласие друг с другом. Исключение составила заметно меньшая экспериментальная ширина полосы отражения у $\mathrm{Al}_{0.15} \mathrm{In}_{0.85} \mathrm{~N} / \mathrm{Al}_{0.2} \mathrm{Ga}_{0.8} \mathrm{~N}$ Б3, что связано, на наш взгляд, либо с флуктуациями толщин и составов эпитаксиальных слоев, либо с упругими напряжениями в Б3, приводящими к изгибу эпитаксиальной структуры в целом.

С помощью моделирования показано, что настройка максимального коэффициента отражения Б3 на желаемую длину волны света является нетривиальной задачей для произвольных распределений показателя преломления на периоде, включая таковые с плавными интерфейсами. Эта настройка требует предварительного расчета спектральной зависимости коэффициента отражения и по ее результатам - коррекции периода БЗ. Ввиду практической важности данной задачи точная настройка может потребовать нескольких итераций.

С помощью моделирования найдено, что переход от ступенчатого профиля к профилям с плавными (градиентными) интерфейсами, нужными для понижения сопротивления Б3, сопровождается уменьшением максимального коэффициента отражения и сужением полосы отражения. Однако эти негативные тенденции могут быть компенсированы за счет увеличения числа периодов в Б3.

Показано, что в случае недостаточно большого числа периодов в Б3, френелевское отражение света на выходном интерфейсе может существенно повлиять на спектральную зависимость коэффициента отражения. 
Проявлениями такого влияния могут стать, в частности, во-первых, значимая асимметрия спектральной зависимости коэффициента отражения относительно настроечной длины волны $\lambda_{0}$, и, во-вторых, заметный уровень отражения света за пределами основной спектральной полосы. Последний эффект в принципе может привести к возникновению паразитной генерации в ВИЛ за пределами полосы отражения Б3. При увеличении числа периодов Б3 это влияние френелевского отражения ослабляется.

\section{Конфликт интересов}

Авторы заявляют, что у них нет конфликта интересов.

\section{Список литературы}

[1] K. Iga. Japanese J. Appl. Phys., 57 (8S2), 08PA01 (2018). DOI: 10.7567/JJAP.57.08PA01

[2] K.J. Ebeling, R. Michalzik. VCSEL Technology for Imaging and Sensor Systems Applications. In: 22nd Microoptics Conf. IEEE, (2017), p. 20-21. DOI: 10.23919/MOC.2017.8244477

[3] E. Watanabe, K. Kodate. Multi-Light Source Compact Optical Parallel Correlator (MLCOPaC) for Facial Recognition Using VCSEL Array. In: 19th Congress of the International Commission for Optics: Optics for the Quality of Life International Society for Optics and Photonics, (2003), p. 208-209. DOI: $10.1117 / 12.523842$

[4] E. Watanabe, N. Takeda, K. Kodate. Fabrication and Evaluation of a Facial Recognition System Based on PJTC Using Two-Dimensional VCSEL Array Module. In: Practical Holography XVII and Holographic Materials IX International Society for Optics and Photonics, (2003), v. 5005 , p. $345-356$. DOI: $10.1117 / 12.473886$

[5] S. McEldowney. U.S. Patent N 8,320,621. Washington, DC: U.S. Patent and Trademark Office (2012).

[6] D.K. Serkland, G.M. Peake, K.M. Geib, R.Lutwak, R.M. Garvey, M. Varghese, M. Mescher. VCSELs for Atomic Clocks. In Vertical-Cavity Surface-Emitting Lasers X. International Society for Optics and Photonics, (2006), v. 6132 , p. 613208 . DOI: $10.1117 / 12.647095$

[7] E. Thrush, O. Levi, W. Ha, G. Carey, L.J. Cook, J. Deich, S.J. Smith, W.E. Moerner, J.S. Harris. IEEE J. Quant. Electron., 40 (5), 491 (2004). DOI: 10.1109/JQE.2004.826440

[8] G. Totschnig, M. Lackner, R. Shau, M. Ortsiefer, J. Rosskopf, M.C Amann, F. Winter. Appl. Phys. B, 76 (5), 603 (2003). DOI: $10.1007 / \mathrm{s} 00340-003-1102-1$

[9] A. Lipson. U.S. Patent N 9,831,630. Washington, DC: U.S. Patent and Trademark Office (2017).

[10] K. Iga. Vertical-Cavity Surface-Emitting Laser: Introduction and Review. In Vertical-Cavity Surface-Emitting Laser Devices (Springer, Berlin, Heidelberg, 2003), DOI: 10.1007/978-3-662-05263-1_1

[11] H. Soda, K.I. Iga, C. Kitahara, Y. Suematsu. Jpn. J. Appl. Phys., 18 (12), 2329 (1979). DOI: 10.1143/JJAP.18.2329

[12] M.H. Crawford. OSA Trends in Optics and Photonics Series, 15, 104 (1998).
[13] T. Sakaguchi, T. Shirasawa, N. Mochida, A. Inoue, M. Iwata, T. Honda, F. Koyama, K. Iga. Highly reflective AlN-GaN and $\mathrm{ZrO} / \mathrm{sub} 2 /-\mathrm{SiO} / \mathrm{sub}$ 2/multilayer Reflectors and Their Applications for InGaN-GaN Surface Emitting Laser Structures. In Conf. Proceedings. LEOS'98. 11th Annual Meeting. IEEE Lasers and Electro-Optics Society 1998 Annual Meeting. IEEE, (1998), v. 1, p. 34-35. DOI: 10.1109/LEOS.1998.737719

[14] D. Kasahara, D. Morita, T. Kosugi, K. Nakagawa, J. Kawamata, Y. Higuchi, H. Matsumura, T. Mukai. Appl. Phys. Express, 4 (7), 072103 (2011). DOI: 072103.10.1143/APEX.4.072103

[15] A.V. Nurmikko, J. Han. Progress in Blue and Near-Ultraviolet Vertical-Cavity Emitters: A status report. In: Vertical-Cavity Surface-Emitting Laser Devices. (Springer, Berlin, Heidelberg, 2003), p. 343.

DOI: $10.1007 / 978-3-662-05263-1 \_11$

[16] S. Arafin, H. Jung. Recent Progress on GaSb-Based Electrically-Pumped VCSELs for Wavelengths Above $4 \mu \mathrm{m}$. In Image Sensing Technologies: Materials, Devices, Systems, and Applications VI, (2019), v. 10980, p. $109800 \mathrm{H}$. DOI: $10.1117 / 12.2522418$

[17] E.F. Schubert, L.W. Tu, G.J. Zydzik, R.F. Kopf, A. Benvenuti, M.R. Pinto. Appl. Phys. Let., 60 (4), 466 (1992). DOI: $10.1063 / 1.106636$

[18] M.G. Peters, B.J. Thibeault, D.B. Young, J.W. Scott, F.H. Peters, A.C. Gossard, L.A. Coldren. Appl. Phys. Lett., 63 (25), 3411 (1993). DOI: 10.1063/1.110156

[19] A. Mutig. Physical Processes in Lasers and VCSEL Design. In: High Speed VCSELs for Optical Interconnects (Springer, Berlin, Heidelberg, 2011)

[20] S.A. Chalmers, K.L. Lear, K.P. Killeen. Appl. Phys. Lett., 62 (14), 1585 (1993). DOI: 10.1063/1.109608

[21] G. Brummer, D. Nothern, A.Y. Nikiforov, T.D. Moustakas. Appl. Phys. Lett., 106 (22), 221107 (2015). DOI: $10.1063 / 1.4922215$

[22] H. Morkoç. Handbook of Nitride Semiconductors and Devices, GaN-Based Optical and Electronic Devices. (WileyVCH, Weinheim: Chichester, 2008), v. 53.

[23] S. Yoshida, K. Ikeyama, T. Yasuda, T. Furuta, T. Takeuchi, M. Iwaya, S. Kamiyama, I. Akasaki. Jpn. J. Appl. Phys., 55 (5S), 05FD10 (2016). DOI: 10.7567/JJAP.55.05FD10

[24] W. Muranaga, T. Akagi, R. Fuwa, S. Yoshida, J. Ogimoto, Y. Akatsuka, S. Iwayama, T. Takeuchi, S. Kamiyama, M. Iwaya, I. Akasaki. Jpn. J. Appl. Phys., 58 (SC), SCCC01 (2019). DOI: 10.7567/1347-4065/ab1253

[25] T. Yasuda, T. Takeuchi, M. Iwaya, S. Kamiyama, I. Akasaki, H. Amano. Appl. Phys. Express, 10 (2), 025502 (2017). DOI: 10.7567/APEX.10.025502

[26] C. Zhang, R. ElAfandy, J. Han. Appl. Sciences, 9 (8), 1593 (2019). DOI: 10.3390/app9081593

[27] J. Chang, D. Chen, L. Yang, Y. Liu, K. Dong, H. Lu, R. Zhang, Y. Zheng. Scientific Reports, 6(1), 1 (2016). DOI: $10.1038 /$ srep29571.

[28] H. Kogelnik, C.V. Shank. J. Appl. Phys., 43 (5), 2327 (1972). DOI: $10.1063 / 1.1661499$

[29] С.Ю. Карпов, С.Н. Столяров. УФН, 163 (1), 63 (1993). DOI: 10.3367/UFNr.0163.199301b.0063. [S.Yu. Karpov, S.N. Stolyarov. Phys. Usp. 36 (1), 1 (1993). DOI: 10.1070/PU1993v036n01ABEH002061] 
[30] Н.Н. Мартынов, С. Н. Столяров. Квантовая электроника, 5 (8), 1853 (1978). [N.N. Martynov, S.N. Stolyarov. Soviet J. Quant. Electron., 8 (8), 1056 (1978).

DOI: 10.1070/QE1978v008n08ABEH010615]

[31] М. Борн, Э. Вольф. Основы оптики (Наука, Гл. ред. Физматлит., 1973)

[32] J. Dorsaz, J.F. Carlin, S. Gradecak, M. Ilegems. J. Appl. Phys., 97 (8), 084505 (2005). DOI: 10.1063/1.1872197

[33] R. Butté, J.F. Carlin, E. Feltin, M. Gonschorek, S. Nicolay, G. Christmann, D. Simeonov, A. Castiglia, J. Dorsaz, H.J. Buehlmann, S. Christopoulos, G. Baldassarri Höger von Högersthal, A.J.D. Grundy, M. Mosca, C. Pinquier, M.A. Py, F. Demangeot, J. Frandon, P.G. Lagoudakis, J.J. Baumberg, N. Grandjean. J. Phys. D: Appl. Phys., 40 (20), 6328 (2007). DOI: $10.1088 / 0022-3727 / 40 / 20 / \mathrm{S} 16$

[34] R. Goldhahn, C. Buchheim, P. Schley, A.T. Winzer, H. Wenzel. Optical Constants of Bulk Nitrides. In Nitride Semiconductor Devices: Principles and Simulation (Wiley Weinheim, Germany, 2007)

[35] База данных показателей преломления („Refractive index database") [Электронный pecypc]. URL: https://refractiveindex.info (дата обращения: 01.05.2019).

[36] C.C. Kao, Y.C. Peng, H.H. Yao, J.Y. Tsai, Y.H. Chang, J.T. Chu, H.W. Huang, T.T. Kao, T.C. Lu, H.C. Kuo, S.C. Wang, C.F. Lin. Appl. Phys. Lett., 87 (8), 081105 (2005).

DOI: $10.1063 / 1.2032598$

[37] W.Y. Lin, D.S. Wuu, S.C. Huang, R.H. Horng. IEEE Transactions on Electron Devices, 58 (1), 173 (2010). DOI: 10.1109/TED.2010.2084579 